\title{
KAROUBI-VILLAMAYOR $K$-THEORY IS NOT HOMOTOPY QUILLEN THEORY
}

JAN R. STROOKER

\begin{abstract}
It is shown that in general the Karoubi-Villamayor group $K^{-2}$ is not a homomorphic image of Milnor's $K_{2}$, establishing the title statement.
\end{abstract}

A functor $F$ from rings (possibly without identity) to groups or abelian groups is called a homotopy functor if the canonical map $F(R) \rightarrow F(R[t])$ is an isomorphism for every ring $R$. To each $F$ one may attach its "homotopization" $H F=\bar{F}$ which is a homotopy functor. There is an epimorphism $F \rightarrow \bar{F}$ (onto for every ring) through which every morphism from $F$ to homotopy functors uniquely factors. For details consult [3], [7] or [6]. Since there are natural transformations from the Quillen-Swan-Gersten $K_{n}$-groups to the Karoubi-Villamayor $K^{-n}$-groups, and the latter are homotopy functors, it is natural to wonder whether they are the homotopizations of the $K_{n}$. This is lent credence by the fact that $K^{-1}=\bar{K}_{1}$ and that for regular rings one even has $K^{-n}=K_{n}$. The following question was therefore raised [7], [2]:

(Q) Are the canonical morphisms of functors $\bar{K}_{n} \rightarrow K^{-n}$ isomorphisms for all $n \geqslant 1$ ?

These maps can also be described as edge homomorphisms in a spectral sequence due to Gersten and Anderson [4], [1].

While we were working on [6], Sharma and I certainly kept (Q) in mind, but could not reach a conclusion. We did however construct a "bad" ring, which demolished another plausible question concerning Karoubi-Villamayor theory. Once Quillen had, shortly thereafter, proved the so-called Fundamental Theorem of algebraic $K$-theory, it was clear that our ring also furnished a counterexample to (Q). We announced this in [9] (the "Autorreferat" of [6]); belatedly, the present note fills in the details.

In explaining the example, I shall adopt the point of view, and freely use the results, of [6]. Let $R$ be a ring with identity. The Fundamental Theorem $[5$, p. 237] asserts there is a split exact sequence

$$
0 \rightarrow K_{n}(R) \rightleftarrows K_{n}(R[t]) \oplus K_{n}\left(R\left[t^{-1}\right]\right) \rightarrow K_{n}\left(R\left[t, t^{-1}\right]\right) \rightleftarrows K_{n-1}(R) \rightarrow 0
$$

Received by the editors April 3, 1978.

AMS (MOS) subject classifications (1970). Primary 18F25, 13 D15.

Key words and phrases. Karoubi-Villamayor and Quillen $K$-theory, Milnor's $K_{2}$, homotopy functors, Fundamental Theorem. 
for $n>1$. Apply the homotopization functor $H$ and bear in mind that $H K_{n}=\bar{K}_{n}$ is a homotopy functor. This gives a split short exact sequence

$$
0 \rightarrow \bar{K}_{n}(R) \rightarrow \bar{K}_{n}\left(R\left[t, t^{-1}\right]\right) \rightarrow \bar{K}_{n-1}(R) \rightarrow 0 .
$$

Now abbreviate the Laurent polynomial functor $-\left[t, t^{-1}\right]$ by $\Lambda$ and the loop space functor $\left(t^{2}-t\right)-$ by $\Omega$ and write $K^{0}$ for $\bar{K}_{0}$. Then $K^{-n}(\Lambda R)=$ $K^{-1}\left(\Omega^{n-1} \Lambda R\right)=K^{-1}\left(\Lambda \Omega^{n-1} R\right)$. The latter group equals $K^{-1}\left(\Omega^{n-1} R\right) \oplus$ $K^{0}\left(\Omega^{n-1} R\right)$ by $\left[6\right.$, p. 88], hence $K^{-n}(R) \oplus K^{0}\left(\Omega^{n-1} R\right)$. By the universality of $H$, we get compatible vertical maps between the two split short exact sequences:

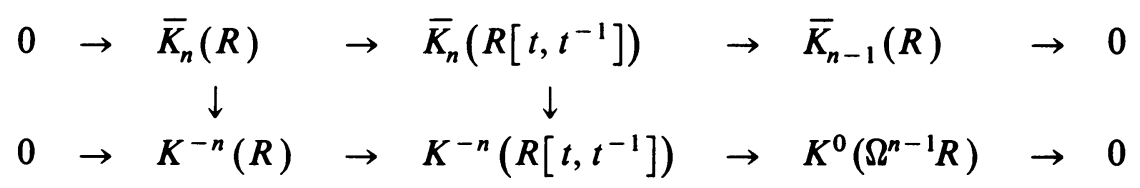

If both maps are isomorphisms, so will be the induced map $\bar{K}_{n-1}(R) \rightarrow$ $K^{0}\left(\Omega^{n-1} R\right)$. For $n=2$ we have $\bar{K}_{1}=K^{-1}$, so it is enough to find a ring $R$ with $K^{-1}(R) \cong K^{0}(\Omega R)$. Such a ring was exhibited in [6, p. 93]: $R=$ $\mathrm{Z}[X] /\left(X^{2}-2^{2 m}\right), m \geqslant 1$. In fact $K^{-1}(R)=\mathbf{Z} /(2)$ while $K^{0}(\Omega R)=\mathbf{Z} /(2) \oplus$ $\mathrm{Z} /(2)$. Therefore $K^{-2}(A)$ is not a homomorphic image of $K_{2}(A)$ for $A=$ $\mathrm{Z}[X] /\left(X^{2}-2^{2 m}\right)\left[t, t^{-1}\right], m \geqslant 1$, and $\bar{K}_{2}(A) \rightarrow K^{-2}(A)$ is not surjective.

\section{REFERENCES}

1. D. M. Anderson, Relationship among $K$-theories, Algebraic $K$-theory. I (Proc. Battelle Inst. Conf., 1972), Lecture Notes in Math., vol. 341, Springer-Verlag, Berlin and New York, 1973, pp. $52-67$.

2. H. Bass, Some problems in "classical" algebraic $K$-theory, Algebraic $K$-theory. II (Proc. Battelle Inst. Conf., 1972), Lecture Notes in Math., vol. 342, Springer-Verlag, Berlin and New York, 1973, pp. 1-70.

3. S. M. Gersten, Homotopy theory of rings and algebraic K-theory, J. Algebra 19 (1971), 396-415.

4. , Higher K-theory of rings, Algebraic K-theory. I (Proc. Battelle Inst. Conf., 1972), Lecture Notes in Math., vol. 341, Springer-Verlag, Berlin and New York, 1973, pp. 3-42.

5. D. Grayson, Higher algebraic $K$-theory. II (after $D$. Quillen), Algebraic $K$-theory (Proc. Evanston Conf., 1976), Lecture Notes in Math., vol. 551, Springer-Verlag, Berlin and New York, 1976, pp. 217-240.

6. P. K. Sharma and J. R. Strooker, On a question of Swan in algebraic K-theory, Ann. Sci. Ecole Norm. Sup. (4) 6 (1973), 85-94.

7. R. G. Swan, Algebraic K-theory, Actes du Congrès International des Mathématiciens (Nice, 1970), Gauthier-Villars, Paris, 1971, pp. 191-199.

8.

9. Zentralblatt für Mathematik und ihre Grenzgebiete, 261 (1974), 108-109, review 18011.

Department of Mathematics, University of Utrecht, 3508 TA Utrecht, The NetherLANDS 